\title{
A Pilot-Scale Study on the Extraction \& Optimization of Keratin from Human Hair - An Adapted Strategy for the Control of Environmental Menace
}

\author{
Punam Sen ${ }^{1^{*}}$, Arun. C. $\mathbf{M}^{2}$, Divvyapriya. $\mathrm{J}^{2}$ \\ ${ }^{1}$ Associate Professor, Department of Microbiology, PSG College of Arts \& Science, Coimbatore-641014 \\ ${ }^{2}$ Department of Microbiology, PSG College of Arts \& Science, Coimbatore-641014
}

\begin{abstract}
Large aggregates of the keratin based pollutants are known to cause intense threat in contaminating the environment of contact region than in the discrete region. Diversifying the application of keratin could be a promising phenomenon for reducing the keratin pollution. Accordingly, the undertaken work was designed to optimize the parameters for the keratin extraction from human hair with a potential source of application in the environment. Sequence of the effective extraction dealt with pre-treatment of human hair with a surfactant (sodium dodecyl sulphate), followed by the digestion of hair with sodium sulfide $\left(\mathrm{Na}_{2} \mathrm{~S}\right)$ at $\mathrm{pH} 12$ - 14 and then stirred with magnetic stirrer at $50^{\circ} \mathrm{C}$. The aliquot was then centrifuged at $\mathrm{pH}$ of $3-4$, following which the precipitated keratin was extracted, dried and pulverized. The conformational study of the extracted keratin was done by performing Ninhydrin test and Fourier transform infrared spectroscopy (FTIR) analysis. The extracted keratin can be exploited in several applications such as, active component in bone replacement, hydro-gel preparation, cosmetics, scaffold preparation, bio degradable films etc. Hence, this work highlighted on the optimal isolation of pure keratin from human hair, paving away the environmental pollutants and advent a healthy grid of societal benefit.
\end{abstract}

Keywords: Keratin, SDS, Filtration, FTIR

\section{Introduction}

Solid waste management of urbanized society has become a recent interest due to dreadful effects caused by the untreated effluents and potential bio-compounds that can be extracted. Keratin waste from various sources like poultry, slaughter houses, leather industry and human hair are known to cause deteriorating effect on human and environment [1]. Being recalcitrant to many simple proteases, keratin when persists in the environment is anticipated to cause long term effects like causing pollution in the environment and imparting diseases like chlorosis and fowl cholera in human [2]. As no data over current keratin production is known, the estimated potential to produce keratin from only $40 \times 10^{6}$ tons of chicken feather [3] and $6.9 \times 10^{5}$ tons of human hair [4] is $4.1 \times 10^{4}$ tons per year (in a rate of $80 \%$ extraction) excluding the leather industry waste, wool waste and other slaughter house waste. Recent trends of sustainable development and wide attraction towards natural protein and its derived materials lead to exploit keratin as reliable source for the day to day application. This super coiled polypeptide with extensive disulfide cross linking is classified into two types soft and hard Keratin, with $1 \%$ and 5\% sulfur content respectively.

Keratin today finds its uses in various fields like cosmetics, Pharmacology, biomedical [5-6] and scaffold

*Corresponding Author: Dr. Punam Sen, Associate Professor, Department of Microbiology, PSG College of Arts \& Science, Coimbatore-641014. Email: punamsen@yahoo.co.in preparation. The keratin extracted from human are found to be more biocompatible, less immune stimulating when used in transplantation and are readily biodegradable [7]. Amidst the substantial development that finds use of keratin into various products like foods, catalysis, bone replacement, cosmetics and fertilizers, still cumbersome keratin finds its way into landfills only due to lack of efficient technology of contaminant free extraction.

The Alkali reduction [8] method which was previously found effective among the rest of available techniques of extraction is employed in this experiment. Current work aims to elevate the process in industrial scale view and optimization aims the utmost productivity of the industry which should be higher than $75 \%$ extraction ratio (maximum achieved till date) [8]. The work here concentrated mainly on to reduce the environmental impact caused by the keratin based waste resources with effective extraction of pure keratin, less labor and production cost and less resources consumption. The commercial form of keratin finds its use as additive in cosmetics, hair care products, sutures, antimicrobial bio-films, and as active component of bone replacement.

Notable role of keratin is centered in cosmetics and wound healing materials and with $50 \%$ share estimating to about 3500 tons of keratin consumption annually, cosmetics highly exploit the keratin resources. Keratin aids in wound healing by directly activating the keratinocytes [9] - skin cells, activating proliferation of the cells in the wounded region. Alpha keratin builds outer skin; aids in hair care, nail growth and beta keratin acts as a precursor for vitamin $\mathrm{A}$, 
hence promoting the eye sight. Amidst raising trends in exploiting keratin into diverse application, the cost of keratin acts as only constrains, so a proper protocol for keratin extraction from available resources can abruptly increase the utilization of the same. Reducing the need of synthetic protein processing by promoting chemical extraction of natural proteins will simultaneously decrease the environmental contamination and increase the exploitation of natural recourses in an economically feasible manner. Commercially, keratin is used in the hair treatment medications, cosmetics, medial application, and as coating in various medical applications like sutures [10].

Keratin from human hair like other mammalian Keratin is $\alpha$-keratin. Previous approach for the extraction of the keratin is found quite less feasible, with application of greater electric and mechanical energy copulation. Works proposed earlier demand the need for continuous digestion of the keratin over the magnetic stirrer[11], which pulls the requirement of huge amount of power consumption [12], [13].The proposed method aims to reduce the contaminant and debris to the maximum extent, ensuring the product stays extra pure. There are very few literatures that are concentrated over the extraction of keratin from human hair, due to unknown notion, this area of research is still found to be least attracted field of study. Moreover, the extraction of keratin from hair is considered a tedious process compared to the extraction of keratin from chicken feather [12], [13], but the current work proposes that the extraction of keratin in much simpler, economic and less time consuming processes comparatively, while accounting the entire process from raw material processing and to the keratin purification.

\section{Materials and methods \\ 2.1 Materials}

Hair Samples (collected from nearby saloon), Sodium Sulfide $\left(\mathrm{Na}_{2} \mathrm{~S}\right)$, Sodium dodecylsulphate (SDS), Over Head Magnetic Stirrer, digestion tank or screw cap beaker, Stirrer, Hot Air Oven.

\subsection{Methodology}

Hair sample was collected from local saloon. The visible debris from the sample were removed and cleaned from the same. $70 \mathrm{~g}$ of the sample taken is soaked in $2 \%$ SDS $(1.5 \mathrm{~L})$ solution for a period of over 20 minutes. The SDS was transferred to another container and can be reused for 4-5 time and the treated hair was washed twice in distilled water. The sample with water was suspended in $1 \mathrm{~L}$ of $1.5 \mathrm{~N} \mathrm{Na} \mathrm{Na}_{2} \mathrm{~S}$ solution, placed in dry region at room temperature for 5 hours and the sample is mixed at $40^{\circ} \mathrm{Cfor}$ a period of $1-2$ hour (in industrial scale the need of magnetic stirrer can be replaced with mechanical agitator which finely mix the hair and produce a complete hydro lysate). The aliquot was centrifuged at $5000 \mathrm{rpm}$ for a period of 5 minutes at room temperature to remove the unwanted debris (which if not removed may lead to wastage of keratin by formation of foam on reaction with the acid added).

The supernatant recovered and the $\mathrm{pH}$ of the solution was brought to 2-3.5 to precipitate out the keratin from the hydrolysate, using $1 \mathrm{~N} \mathrm{HCl}$. The solution was kept undisturbed for 2 hours in order to precipitate out the keratin in white aggregates. Later, the Keratin was filtered from the precipitate and dried at $45^{\circ} \mathrm{C}$, for 2 hours,following which flakes of keratin was obtained. Then, keratin is recovered and pulverized. The significance of work lies in optimizing the parameters for potential extraction of keratin lies in the optimization of parameters controlling the rate and quality of reaction. For this purpose, there were certain comparisons made over the working protocol, that aimed to demonstrate and point out the necessary steps required and eliminate the unwanted steps in order to optimize production. Parameters for the comparison included effects of pretreatment of raw material, need for effective $\mathrm{pH}$ maintenance of the hydro lysate, effect temperature exposure. Keratin obtained was analyzed for purity and quantified. It was subjected to Ninhydrin Test and FTIR analysis, which revealed the amino acid content in the keratin alongside confirming the presence of keratin qualitatively and analytically. The dry weight of the hair sample and the extracted keratin was measured for calculating the efficiency of extraction. In Ninhydrin test [14], copper sulphate solution and potassium hydroxide taken at $1 \%$ each and equal volume of the corresponding solution were mixed for about $5 \mathrm{ml}$, and to the taken solution, $5 \mathrm{ml}$ of Keratin solution was added ( $1 \mathrm{~g}$ keratin in $2 \mathrm{ml}$ of $\mathrm{NaOH}$ ).

\section{Results and Discussion}

The keratin extracted with the optimized criteria, found to be promising and efficient, with maximum extraction of the protein in the given quantity of hair sample. The qualitative and quantitative results of total percentage of keratin extracted are detailed in following result.

\subsection{Effect of pretreatment}

The hair pretreated with SDS (detergent and surfactant) is found to be contaminant free, while the other protein extracted without pretreatment are mixed with contaminant and lots of debris though previously washed with water. The color of keratin is a good indicator of the purity of the keratin, a slight milky grayish shade during recovery, which decolorized to milky yellow after complete dehydration process. These results are comparable with the existing research literatures [3], [6], which supports that the color of keratin is milky yellow. These results also correspond with keratin extracted from various other raw materials like Chicken feather and pig hair. Recording this result could potentially mark further research works handy. The keratin extracted without pretreatment are found to be blackish grey color, where protein at this extract was found to be inseparable from debris. The hydro lysate when treated to reduce the $\mathrm{pH}$ if untreated then would result in foam formation upon reaction with acid, resulting in the wastage of keratin.

\subsection{Optimum pH maintenance}

Hair keratin dissolves and gets hydrolyzed only in the alkaline condition and is non-reactive in neutral and acidic $\mathrm{pH}$. Hence, maintaining a $\mathrm{pH}$ range of 10.5 to 12 would bring out the efficient hydrolysis of the keratin. Highly alkaline $\mathrm{pH}$ causes the structural damage to the keratin and bring out the altered conformational skeleton. $\mathrm{pH}$ maintenance during recovery of the keratin from the hydro lysate was effective in the extraction of the keratin without contamination. It was found that keratin gets precipitated around the $\mathrm{pH}$ range of 2-3 (acidic).

\subsection{Influence of temperature over the keratin extraction}

Temperature applied over the process of extraction of the keratin played a significant role in the extraction procedure. Initially during the digestion of sample, an ambient temperature around $45^{\circ} \mathrm{C}$ to $58{ }^{\circ} \mathrm{C}$ were found to be effective, since the temperature more than the optimum was known to alter the side chain of the keratin sometimes leading to degradation [7]. The observed temperature dependence contradicts the previous literature observations which insisted on input of temperature of at least $70^{\circ} \mathrm{C}$ [15]. The temperature of less than the given, if applied, known to increase the 
duration of extraction process, which can add up to the excessive use of electrical and mechanical consumption. But the undergone work revealed that $50^{\circ} \mathrm{C}$ to $58^{\circ} \mathrm{C}$ was ambient for highest productivity, provided that the initial incubation period is $3-4$ hours (undisturbed). The other place where the heat found its importance was in the drying of keratin during the post extraction process, where exposure of keratin extracted and washed were dried at $45{ }^{\circ} \mathrm{C}$ for a period of 5 hours.

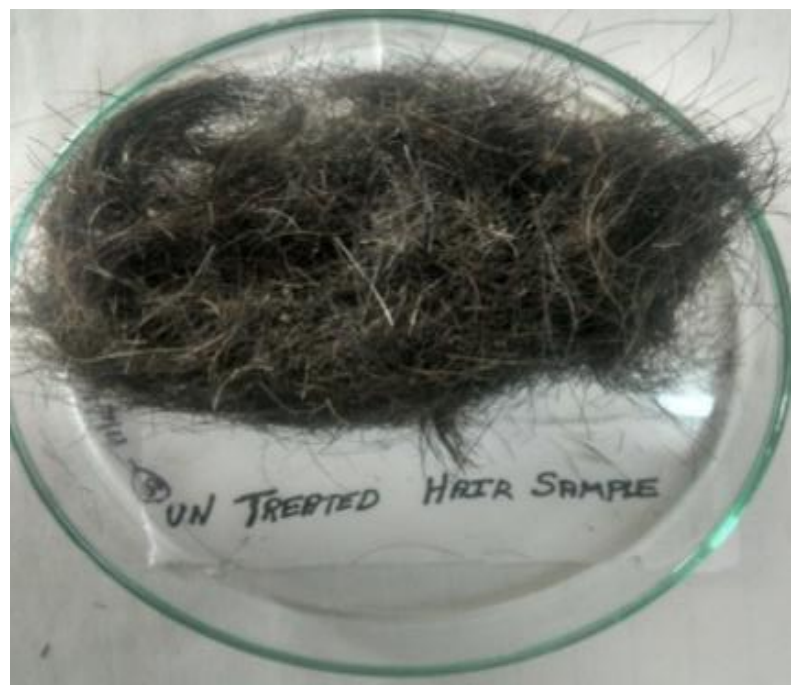

(a) Untreated Hair

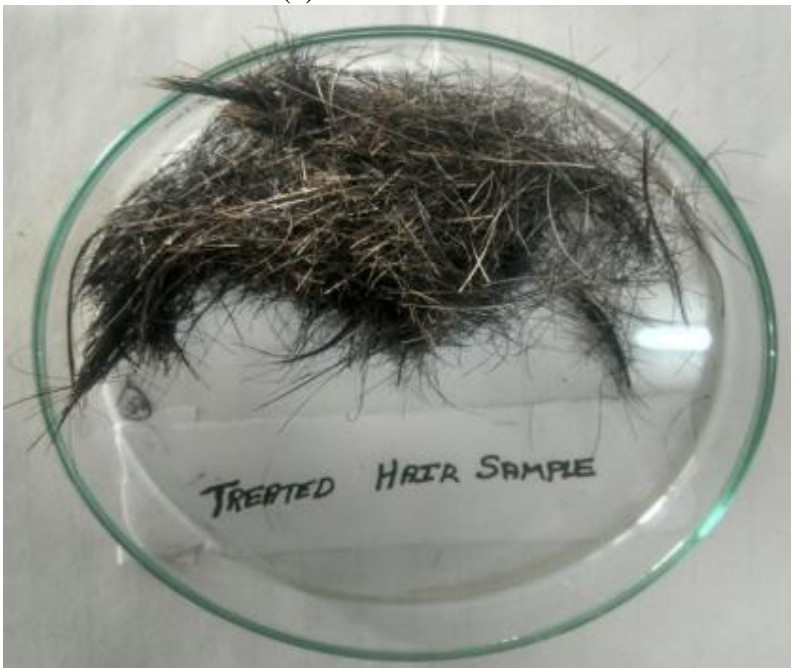

(b) Treated Hair

Figure 1: Treated and untreated hair

\subsection{Post extraction Centrifugation}

Comparable to pretreatment the post extraction centrifugation of the hydro lysate for a period of 10 minutes at $10000 \mathrm{rpm}$ in room temperature would result in effective keratin extraction. Even these debris if not removed may result in minimal foam formation and can add up to the pigmentation of the final keratin extract [10]. Instead of centrifugation, membrane filters too can be substituted for the effective removal of debris allowing only the hydro lysate to be separated.

\subsection{Keratin Extracted (Quantitative)}

Out of $10 \mathrm{~g}$ of the hair sample, a total of $7.28 \mathrm{~g}$ dry weight of the keratin was extracted which accounted for about $73 \%$ of the total dry weight of the human hair sample taken. The color of the extracted keratin was found to be either pale milky yellow or pale milky grey shade.

\subsection{Ninhydrin Test}

The keratin when added to Ninhydrin reagent, the solution turned into deep blue to violet color indicating the presence of the protein. This result corresponds to standard coloration of protein extract [14]. The crude protein with contaminant will not produce a significant coloration and thus promoting false results. So Ninhydrin test being primary analysis contamination in extraction would result in misinterpretation of product obtained, thus a care must be taken and a contamination free extraction must be ensured.Figure4 represents the observed result of Ninhydrin test.

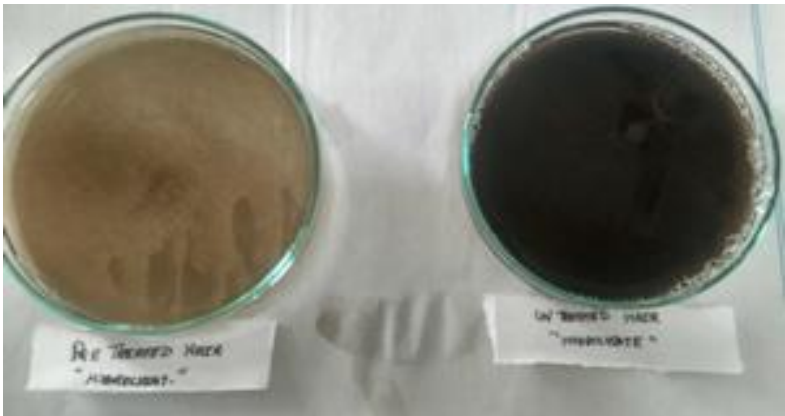

Figure 2: Difference between centrifuged sample (left) and uncentrifuged Hydro lysate sample (right)

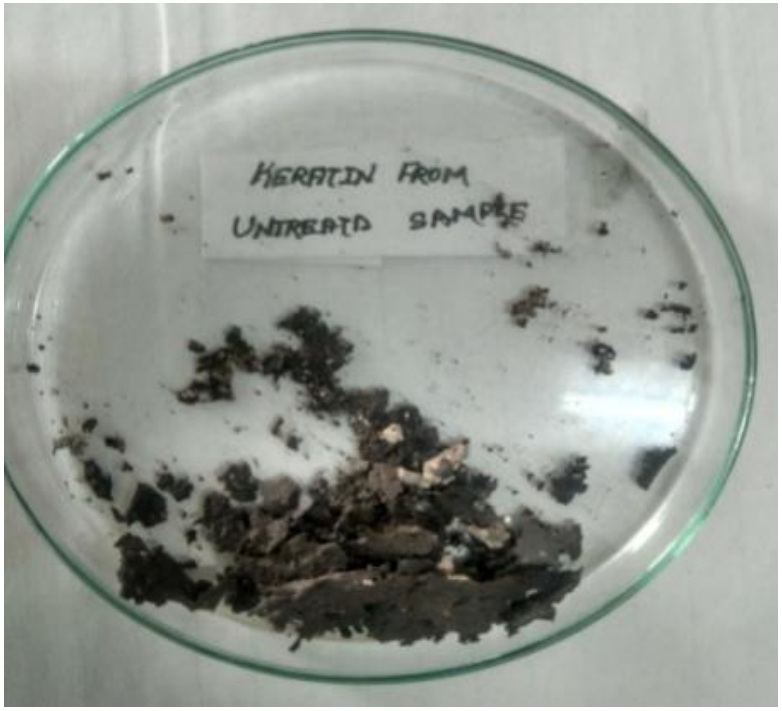

(a) Untreated Keratin

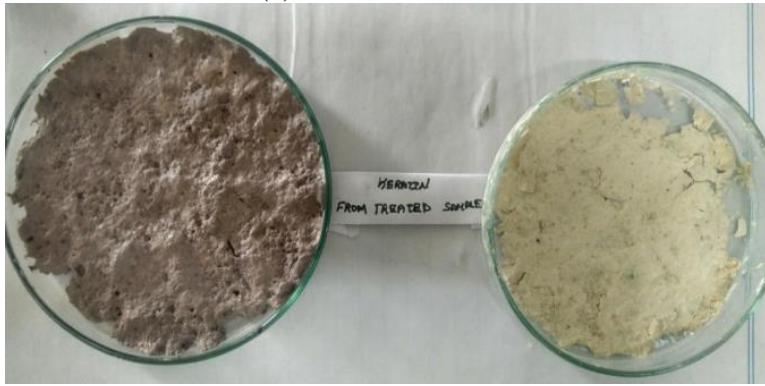

b) Un-centrifuged pretreated sample (LEFT) and completely Treated Keratin (RIGHT)

Figure 3: Extracted Keratin 


\subsection{FTIR Analysis of the Keratin}

FTIR analysis aids in identification of types of compounds present and especially the protein types based on the functional group present in them [16], [17]. FTIR results show that the peaks correspond to the keratin of the standard chart and previous results of keratin [17]. The Figure 5 represents the FTIR analysis of pure pale yellow keratin sample effectively extracted from the experiment conducted.

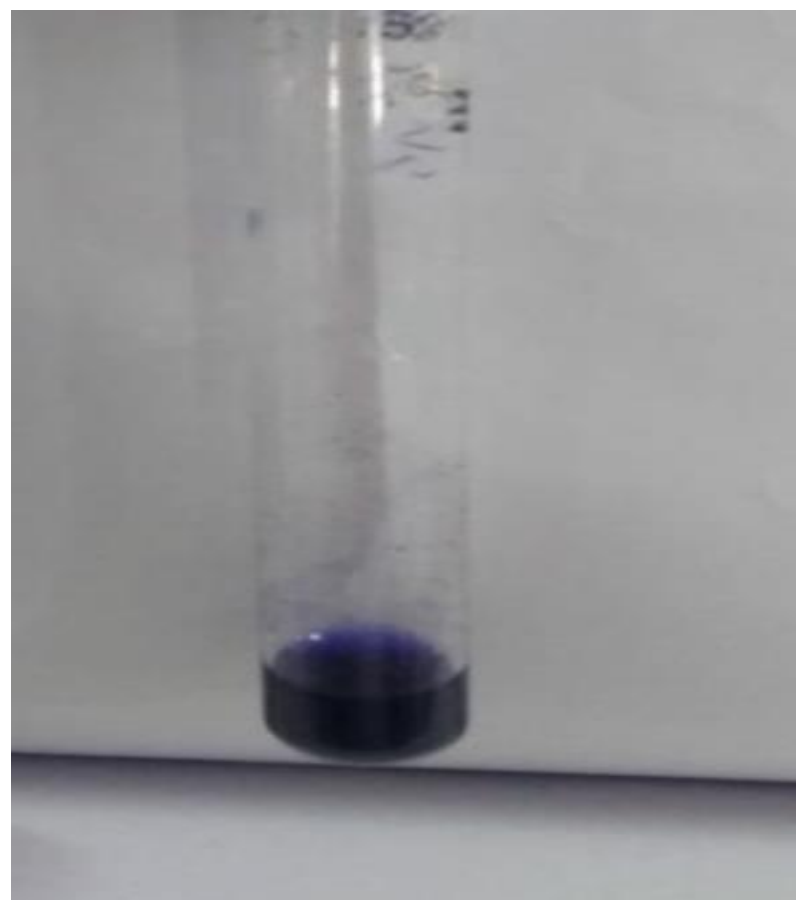

Figure 4: Ninhydrin Test Result (Positive)

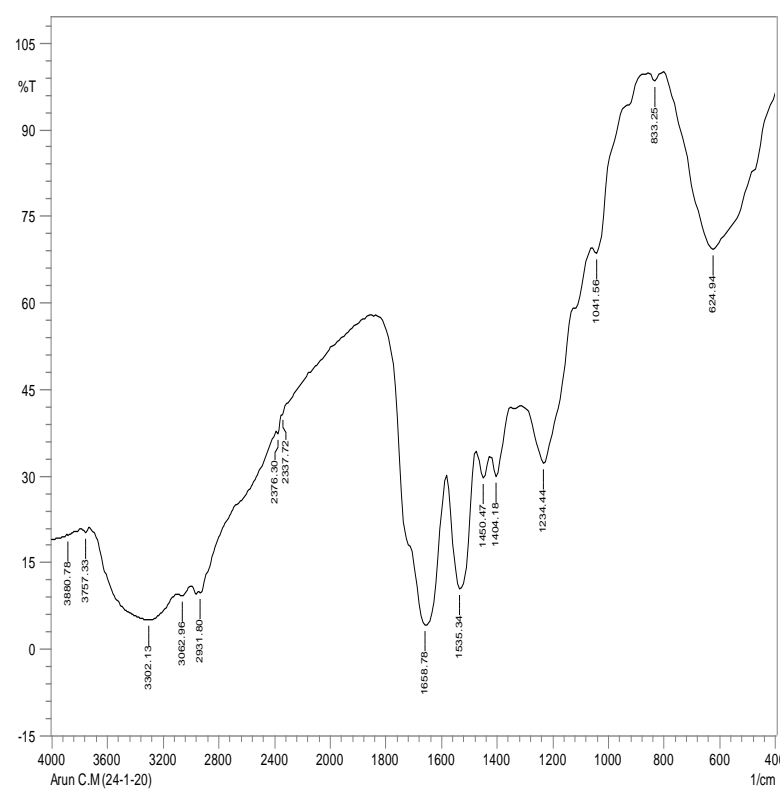

Figure 5: FTIR Results obtained from final Keratin Powder

The peaks correspond to various functional group, the peak at $3757 \mathrm{~cm}^{-1}$ indicates the presence of $\mathrm{H}_{2} \mathrm{O}$ molecules, $3307.13 \mathrm{~cm}^{-1}$ corresponds to $\mathrm{O}-\mathrm{H}$ bonds in carboxylic acids and derivatives, alcohols and phenols and 3062.96 indicates the presence of $\mathrm{C}-\mathrm{H}, \mathrm{CH}_{2}, \mathrm{C}=\mathrm{C}$ alkenes. The peak ranges 2931.80, 2376 and 1668.78 represent C-H Systemic stretch of $\mathrm{CH}_{2}$ and or at fatty acids, systemic stretching vibrations of lipid acyl $\mathrm{CH}_{2}$ groups and $\mathrm{NO}_{2}$ bonding in nitro compounds, Amide I band components of beta pleated structure of protein.
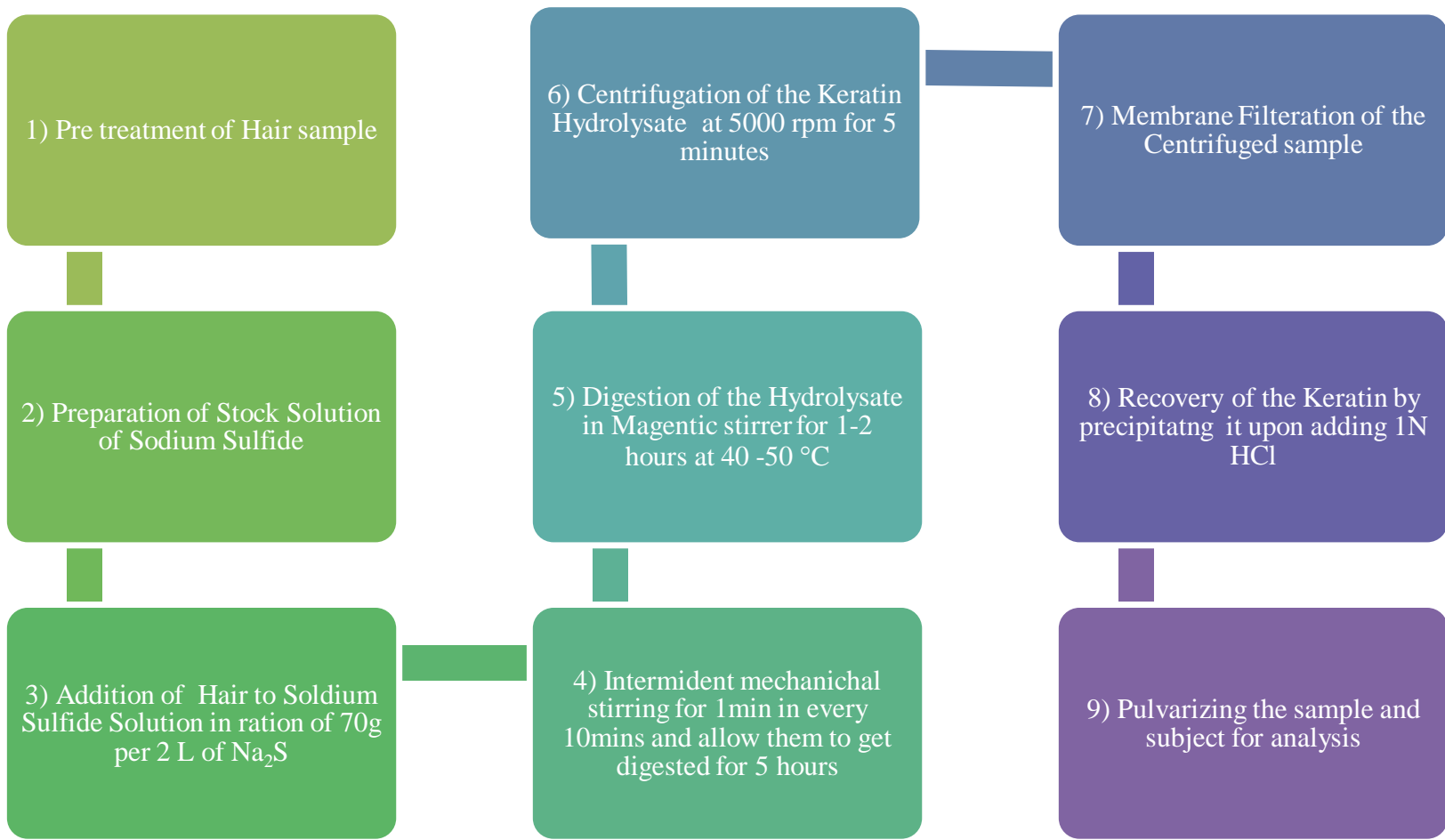

Figure 6: Keratin Extraction technique - flow chart 


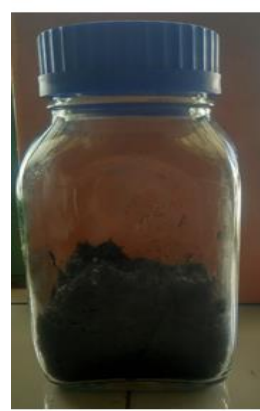

STEP - 1

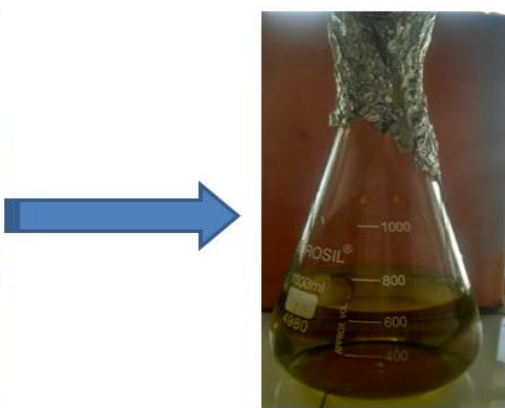

STEP - 2

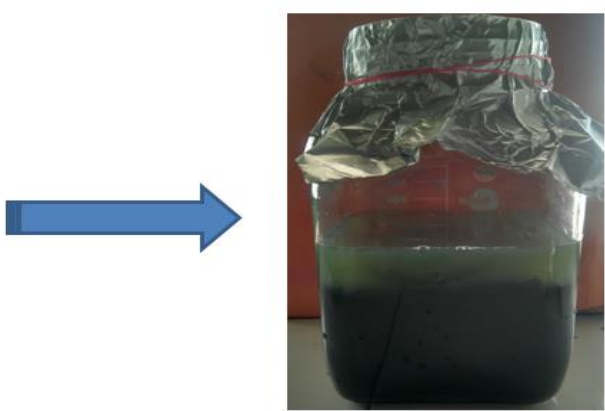

STEP - $\mathbf{3}$

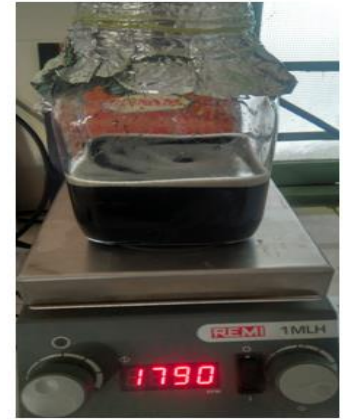

STEP 4 \& 5

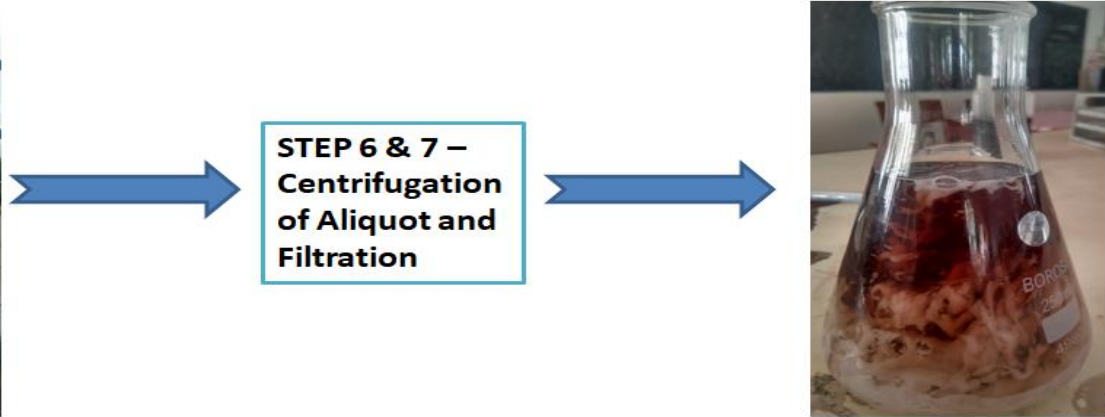

STEP - 8

STEP 9

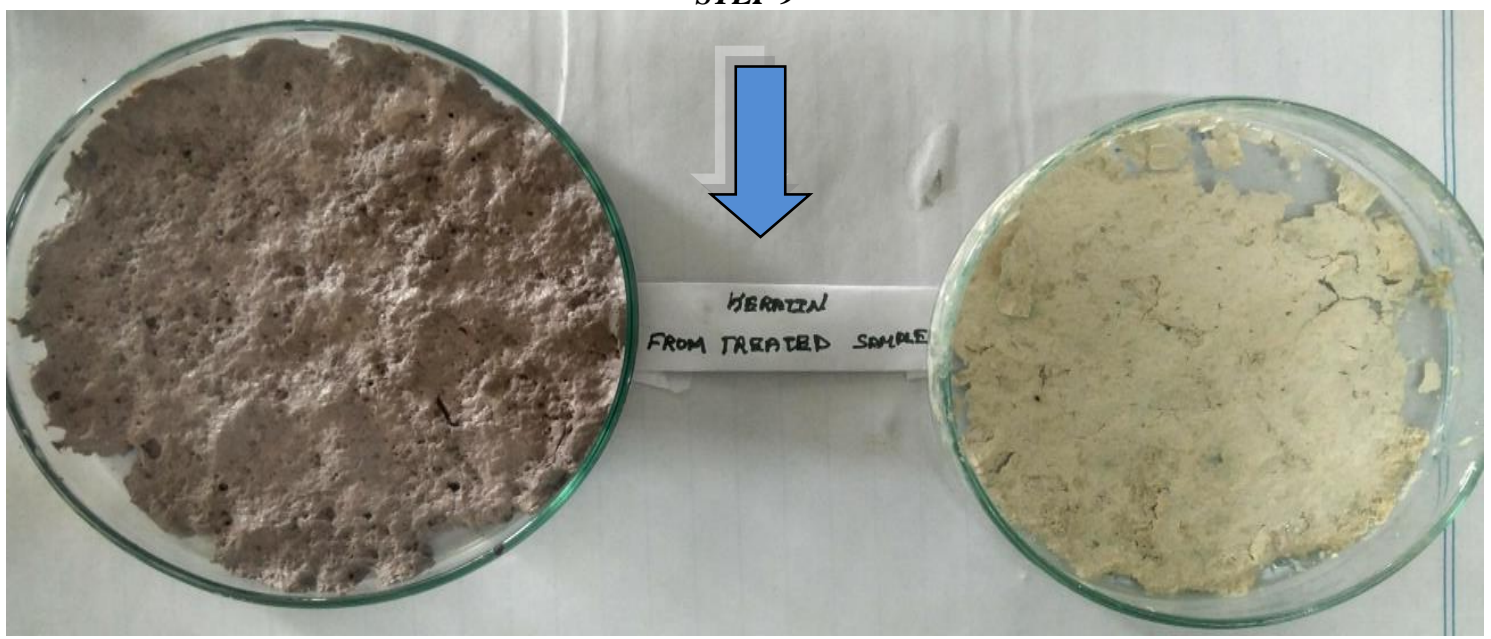

Figure 7: Methods involved in keratin extraction from human hair

The region at 1234.44 represents, Amide III band components of proteins, C-N stretching vibrations from amines, from free amino acids and $\mathrm{P}=\mathrm{O}$ asymmetric stretching of $\mathrm{PO}$-, phosphodiesters. A slight peak at 1176 and distinctive peak at 1041 indicates $\mathrm{C}-\mathrm{O}, \mathrm{C}-\mathrm{C}, \mathrm{C}-\mathrm{N}$, stretching, C-O-H, C$\mathrm{O}-\mathrm{C}$ deformation of carbohydrates and carbohydrate glycoside bonds, this infers the $\mathrm{C}=\mathrm{O}$ glycol protein. 833.24 peak represents S-OR esters out of plane bending, $\mathrm{NO}_{2}-, \mathrm{NO}_{3}$ - and $\mathrm{CO}_{2}$ groups. The final peak at 624.95 infers $\mathrm{C}-\mathrm{H}$ deformation, $\mathrm{SO}_{4}{ }^{2-}, \mathrm{NH}_{2}$ and $\mathrm{NH}$ groups. The peaks fluctuate and many minor peaks seen near the major peeks are due to the fact that the occurrence of hair from different age groups, since the peaks of keratin of different age groups differs feebly [18].Keratin after extraction exhibits wide range of pigmentation between a band of grey white, grey black or milky yellow, but Pure keratin is in milky grey white and milky yellow in color.

The extracted keratin may be highly pigmented due to the presence of two major of keratin in demand. Less importance is staged over such specific downstream processing of extracting less pigmented keratin. In the future research upon thawing light over the area could lead to customize pigments, Eumelanin (Dark) and Phaeomelani [19] (Light) which can be removed or retained according to the grade.

\subsection{Quantity of Keratin extracted}

Upon optimizing the above parameters and precisely conducting the extraction process with no fluctuations from the above mentioned protocol, the quantity of keratin obtained was $7.28 \mathrm{~g}$ per 10 gram of human hair, where the maximum possible theoretical keratin value is $9 \mathrm{~g}$ of keratin per $10 \mathrm{~g}$ of 
human hair sample (Estimating an average of $90 \%$ keratin content). This is estimated to be about $79-80 \%$ of total keratin in human hair, and accounts approximately $73 \%$ in dry weight of dry hair sample. This method finds promising than the previous results of $75 \%$ [8]. Apart from this, the quantity of keratin obtained from poorly treated and contaminated substrates in its crude form weighed around $7.3 \mathrm{~g}$ (treated hair sample with poor centrifugation) and $7.9 \mathrm{~g}$ (untreated hair sample and improper centrifugation) where impurities accounting for about 6 percent of total dry weight, with dark pigmentation making the extract unfit for commercial consumption. These parameters are not discussed in detail among any parallel research papers, thus these results could significantly influence the future research works.

\section{Conclusion}

The undergone work clearly revels that this protocal can effeciently be adapted as scale up strategy. This inferance is supported by following observation and outcome of the result. The observed output of $79 \%-80 \%$ extation is the highest ever, surpassing the previous litrature claim of $75.3 \%$ with whooping difference [8]. Moreover the waste aliquotes released after every stage of keratin extraction can be effectively used as liquid fertilizer, thus making the process completely ecofriendly [20]. Scaling up this treatment process along with proper effluent treatment techniques would promote a complete ecofriendly manufacturing unit. Every experimental results till date conclude that the keratin extraced from human is more biocompatable and its immunogenic effect is negligible [21]. The extracted keratin can be potentially used as biofertilizer in a considerable quantity where the keratin in par with acting as nutrient enrichment, it also exhibits bioremidiating property. The significance of work lies with the raw material of the undergone work, many of these results are obtained previously but not with the human hair instead many similar results were seen in chicken feather and wool, there could be two possibilities in which there was no previous such studies in detail, or these observations might not been previously found significant. The pigmentation of the extacted keratin can excert a considerable thrust over the quality of keratin, and the undergone work revelaed a significant result that majority of pigmentation is due to dirts and poor work hygiene. Pale (milky) yellow is a measure of pure keratin and color in range between very light grey to pale yellow are considered pure which is also confirmed from other studies [22], [23]. Though these results are oriented with human hair keratin extraction, the horizon of application can be extended to treat chicken feather and wool the parallel keratenous wastes, to maximize the production. We could expect much similar positive results in near future with undergone eexperimet by applying over various keratenous wastes dumped in the environment.

\section{Acknowledgment}

We are thankful to the host Institution PSG College of Arts \& Science, Coimbatore, for providing us with all the facilities \& support during the course of work.

\section{Competing interests}

We declare that there is no conflict of interest that would prejudice the impartiality of this scientific work.

\section{Authors' contribution}

All authors of this study have a complete contribution for data collection, data analysis and manuscript writing.

\section{References}

1. Tarun Kumar Kumawat, Anima Sharma, Vishnu Sharma and Subhash Chandra, In Keratin: Keratin Waste: The Biodegradable Polymers, Keratin, MiroslavBlumenberg, IntechOpen, December $19^{\text {th }} 2018,10.5772 /$ intechopen.79502.

2. Williams CM, Lee CG, Garlich JD, Shih JCH. Evaluation of a bacterial feather fermentation product, feather-lysate, as a feed protein. Poultry Science. (1991); 70(1), pp.85-94

3. Debananda Singh Ningthoujam, KeishingTamreihao, Saikat Mukherjee, RakhiKhunjamayum, Laishram Jaya Devi and Roshan Singh Asem, Keratinaceous Wastes and Their Valorization through Keratinolytic Microorganisms, KeratinMiroslavBlumenberg, Intech Open, (2018), 10.5772/intechopen.80051

4. Gupta, Ankush. Human Hair "Waste" and Its Utilization: Gaps and Possibilities. Journal of Waste Managment. (2014). $10.1155 / 2014 / 498018$

5. Reichl S., Film based on human hair keratin as substrates for cell culture and tissue engineering, Biomaterials, (2009); Vol. 30(36), Pp. 6854-6866.

6. Xiao-Chun Yin, Fang-Ying Li, Yu-Feng He, Yan Wang and RongMin Wang Study on Effective Extraction of Chicken Feather Keratins and their films for controlling Drug release, BiomaterialsScience, (2013); Vol.1, Pp. 528-536,

7. Lee, H., Noh, K., Lee, S.C. et al. Human hair keratin and its-based biomaterials for biomedical applications. Tissue EngRegenMed (2014) Vol.11, pp. 255-265.

8. Jiang Shui-qing, Zang Lin, Wu Haixia\& Chi Gang, Study on effective extraction of keratin from human hair wastes. Journal integrated Ferroelectics, (2017) pp.102-107.

9. Mokrejs P, Hutta M, Pavlackova J, Egner P, Benicek L, The cosmetics and dermatological potential of Keratin hydrolysate. J.CosmetDermatol.(2017);Vol.16(4), pp. e21-e27.

10. Sharma, Swati, \& Gupta, Arun., Sustainable Management of Keratin Waste Biomass: Applications and Future Perspectives. Brazilian Archives of Biology and Technology, 59, e16150684. Epub(2016). Pp. 1-14.

11. Wang, Kui\& Li, Rong\& Ma, Jihong\&Jian, Yingkai\&che jiangning., Extracting Keratin by using L-cysteine, GreenChem, (2016); Vol.18,Pp.476-481

12. Pourjavaheri, Firoozeh\&Mohaddes, Farzad\& Shanks, Robert \&Czajka, Michael \& Gupta, Arun.. Effects of Different Purification Methods on Chicken Feather Keratin. Advanced Materials Research, (2014) pp. 941-944.

13. Millington. K.R, Ripon. J.A, Wool as high performance fiber, Structure and Properties of High Performance Fabrics. Woodhead Publishing Series in Textiles (2017); Pp. 367-408

14. Mendel Friedman, L.David Williams, The reaction of Ninhydrin with Keratin proteins. Analytical Biochemistry, (1973), pp. 333345

15. Berhanu, Taame.,Cationization of Cotton Using Extracted Keratin from Human Cut Hair Waste for Salt Free Dyeing With Reactive Dye, International Journal of Scientific \& Engineering Research, (2018) Vol. 9, pp. 907-927..

16 Suren A Tatulian, FTIR Analysis of proteins and proteinMembrane Interactions, Lipid - Protein interactions,(2019) pp. 281-325.

17. Huayan Yang, Shouning Yang, Jilie Kong, Aichun Dong and Shaoning $\mathrm{Yu}$, Obtaining information about protein secondary structures in aqueous solution using Fourier transform IR spectroscopy, Nature Protocols,(2015) pp. 382-396

18. Mohd Abdul Mujeeb and M. K. M Zafar, FTIR Spectroscopic Analysis on Human Hair. International Journal of Innovative Research in Science, Engineering and Technology, (2017) Vol. 6(5), pp. 9327 to 9332

19. Borges. C.R, Roberts. J.C, Wilkins. D.G, Rollins de. Relationship of melanin content: Application to human hair, Analytical Biochemistry, (2001); Vol. 290 (1), Pp. 116-125.

20. GayathriUnnikrishnan, VijayaraghavanRamasamy, Extraction of keratin from Human Hair with production of biofertilizer from waste liquid of hair extraction and its efficient Application on growth yield of Abelmoschusesculentus L. Asian Journal of Biological and Life Sciences, (2020), pp.119-128,

21. Lee, Hanna \& Noh, Kwantae\& Lee, Sang \& Kwon, Il Keun\& Han, Dong-Wook\& Lee, In-Seop\& Hwang, Yu-Shik., Human 
Hair Keratin and Its-Based Biomaterials for Biomedical Applications (2014). Tissue Engineering and Regenerative Medicine. Vol 11, pp. 255-265.

22. Fujii, Toshihiro \& Tamura, Tomohiro \& Hayashi, Kaori \& Ito, Yumiko., Dyeing and Discoloration of Human Hair Keratin Film Treated by Oxidative Hair Color, Journal of Fiber Science and Technology. (2016) Vol 72, pp. 96-103

23. Agarwal, Vipul \& Panicker, Arpana \& Indrakumar, Sushma \& Chatterjee, Kaushik, Comparative study of keratin extraction from human hair. International Journal of Biological Macromolecules (2019), Vol 133. Pp. 382-390 